\title{
Magnetoresistance Calculations for a Two-Dimensional Electron Gas with Unilateral Short-Period Strong Modulation
}

\author{
Karel Výborný \\ I. Institute of Theoretical Physics, University of Hamburg, Jungiusstr. 9, D-20355 Hamburg, Germany \\ Ludvík Smrčka \\ Institute of Physics, Academy of Sciences of the Czech Republic, \\ Cukrovarnická 10, CZ-16253 Praha, Czech Republic \\ Rainer A. Deutschmann \\ Walter Schottky Institut, Technische Universität München, D-85748 Garching, Germany
}

\begin{abstract}
The linear response theory is used to describe magnetoresistance oscillations of short-period unilateral superlattices with strong modulation (or alternatively arrays of coupled quantum wires). The semiclassical description of this system fails for strong magnetic fields (magnetic breakdown) and we employ a simple fully-quantum-mechanical tight-binding model in conjunction with Kubo's formula instead. The resulting magnetoresistance data nicely compare to the experiments while the model opens good intuitive insight into the effects taking place in the system.

PACS numbers: $73.63 . \mathrm{Nm}$
\end{abstract}

\section{INTRODUCTION}

Transport properties of two-dimensional electron systems (2DES) with unidirectional periodical modulation have been studied for more than ten years. Lot of attention has been paid to the case of weak modulation by electric and magnetic fields. Such a system was first prepared by means of holographic techniques by Weiss et al 1 and showed the commensurability oscillations in magnetoresistance for low magnetic fields and Shubnikov-de Haas ( $\mathrm{SdH}$ ) oscillations for high magnetic fields. Periodicity of the former ones can be well understood even in a semiclassical (SC) concept considering the drift of the cyclotron orbit centre in crossed electric and magnetic fields. This approach can also give some quantitative predictions for the magnetoresistance 2. An alternative formulation of the $\mathrm{SC}$ approach 3 relying on the breakdown probability (tunnelling between two closed SC orbits) oscillations is also possible. Gerhardts et al. agonalised the quantum-mechanical (QM) Hamiltonian (and employed the Kubo formula to compute conductivity) finding the oscillating width of Landau bands (Landau levels broadened by the weak modulation into narrow cosine-like bands) to be the basic cause of the effect within the QM picture. QM approach was also applied by Vasilopoulos et al.5.

The SdH oscillations follow naturally from the QM concept owing to the quantization of free electron motion in magnetic fields (Landau levels, LLs). This quantization has to be ad hoc assumed in the SC picture but once this is accepted, the SC theory provides a sufficient description in this case.

Experiments on gated structures manufacture by lithographic techniques performed by Beton et al.6 al- lowed for investigating effects of stronger modulation. Compared to the previously mentioned experiments, the major effect was the quenching of the commensurability oscillations. The SC approach 3 was applicable againd, although quantum calculations were in better agreement with experiments. Later, the SC theories have been extended in order to account for anjptropic scattering 8 and for mofulation by magnetic field 10 (extending the older works211). For QM approach to modulation by magnetic field see e.g.12. All together, the SC approach proved itself to be suitable in these cases.

More recently, samples with modulation potential due to MBE-grown structure were prepared (using cleaved edge overgrowth technique) by Deutschmann et al.13 In contrast to all former experiments, the modulation period $d=15 \mathrm{~nm}$ was shorter by almost one order of magnitude. Owing to this fact and also due to strong modulation (Fermi energy $E_{F} \simeq 4|t|$, where $t$ is the hopping integral between ground states in two neighbouring wells of the potential modulation), the lowest modulation miniband is well separated from higher minibands (condition for this is $d \lesssim \sqrt{3 h^{2} /(2|t| m)}$ ). The magnetoresistance oscillations measured when the Fermi level lies between the modulation bands can be explained by no semiclassical model unless tunnelling betweep open trajectories is assumed (the breakdown formalism 3 mentioned above is necessary at this place). It is thus appropriate to revert to a quantum mechanical description. Moreover, the miniband structure is simple now and allows thus for a good insight into the physics both on the $\mathrm{SC}$ and quantum-mechanical level. 


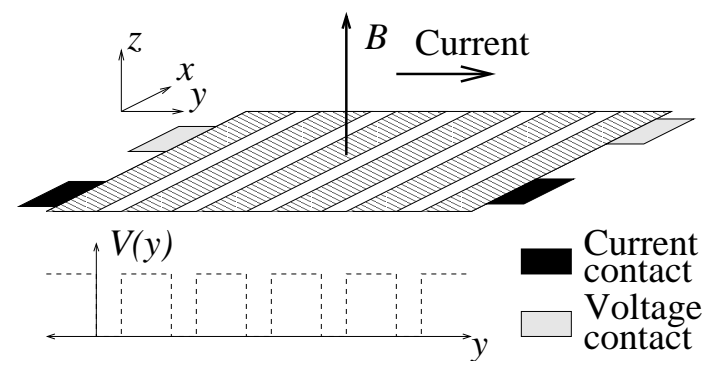

FIG. 1: 2DES with lateral periodical modulation and the effectively two-point-contact geometry.

\section{A. The System and Outline}

In this paper, we refer to experiments carried out on GaAlAs/GaAs structures first reported in 13 , see also more detailed description in 14.15 . These are superlattices with strong unilateral short-period (electric-field) modulation. The substantial difference to previous studies (see the Introduction) is the shortness of the modulation period: only the lowest modulation band (and not many of them) is occupied under those circumstances which makes the usage of quantum mechanics unevitable. Moreover, absence of the higher modulation bands makes the model very transparent. We concentrate on magnetoresistance measurements at different concentrations of electrons (which could be varied by a gate voltage over the range $0.5 \div 5.0 \times 10^{11} \mathrm{~cm}^{-2}$ ). The system is sketched in Fig. 11.

The band structure in zero magnetic field calculated within the Kronig-Penney model can be well approximated by

$$
E\left(k_{x}, k_{y}\right)=\frac{\hbar^{2} k_{x}^{2}}{2 m}-2|t| \cos k_{y} d
$$

with $4|t| \approx 3.8 \mathrm{meV}$ and $m$ equal to the effective mass of electrons in GaAs. The next modulation band is well above the Fermi level $E_{F}$ for all accessible concentrations.

If the Fermi level lies near the bottom of the band $\left(-2|t|<E_{F} \ll 2|t|\right)$, the system resembles a free 2DES (i.e. paraboloidal spectrum with modified effective mass in the modulation direction $m_{y}=\hbar^{2} / 2|t| d^{2} \approx 2.7 m$ ). If the Fermi level lies high above the modulation band edge $\left(E_{F} \gg 2|t|\right)$, the dispersion relation is like the one of an array of separated one-dimensional wires ( i.e. parabolic across $k_{x}$ and nearly constant along $k_{y}$ ) while the deviation from $E=\hbar^{2} k_{x}^{2} / 2 m$ reflects the coupling of wires.

The structure of this paper is the following. At the beginning we will review the semiclassical approach as a favourite tool used to describe magnetotransport experiments. We will also compare the zero-field density of states (DOS) with the $B \neq 0$ DOS computed in Sec. II]. It will give us illustrative examples of a situation when the SC theory is expected to be successful and of another situation when it should fail. The condition of applica- bility of the SC approach will be shown to be $\hbar \omega_{\text {eff }} \ll 2|t|$ whereas $\omega_{\mathrm{eff}}=e B / m_{\mathrm{eff}}=e B / \sqrt{m m_{y}}$.

In the second part (Sec. II) we will describe a fullyquantum-mechanical one-particle model and demonstrate that the gaps in the DOS emerging from this model coincide with extrema in the measured magnetoresistance. Then we will employ the linear response theory (section III) in order to calculate the magnetoresistance and we will compare it with the experiments.

\section{B. Semiclassical Approach}

The physical quantity of central importance in transport theories is the density of states $g(E)$ (DOS) at the Fermi level $E_{F}$. It is known that its structure reflects features of the resistance (both as a function of $B$, for instance) but the relation between these two quantities is not simple.

The SC theory attempts to explain the behaviour of electrons subject to a magnetic field in terms of the zerofield Fermi surfaces. We demonstrate that the zero-field DOS plus an extra quantization condition is a fairly good approximation to the realistic DOS (computed by our model, from Section III) at low magnetic fields $\left(\hbar \omega_{\text {eff }} \ll\right.$ $2|t|)$. However, there is a drastic difference between these two densities of states for high magnetic fields indicating failure of the SC theory (see Fig. 3).

Let us briefly review the SC approach suggested by Lifshitz and Onsager (see e.g.16). We construct the Fermi contour $E_{F}=E\left(k_{x}, k_{y}\right)$ for a given Fermi level. The statements are that (1) the Fermi contour rotated by 90 degrees and scaled by $\ell^{2}=\hbar / e B$ corresponds to the real-space trajectory of an electron (see Fig. 2) and (2) if the contour is closed, then it is allowed only if the magnetic flux passing through the area enclosed by the real-space trajectory is an integer multiple of the magnetic flux quantum $\Phi_{0}=h / e$. In the QM picture, this quantization condition corresponds to the situation when $E_{F}=\hbar \omega_{\text {eff }}\left(n+\frac{1}{2}\right)$ for some integer $n$, i.e. when the $n^{\text {th }}$ LL passes through the Fermi level.

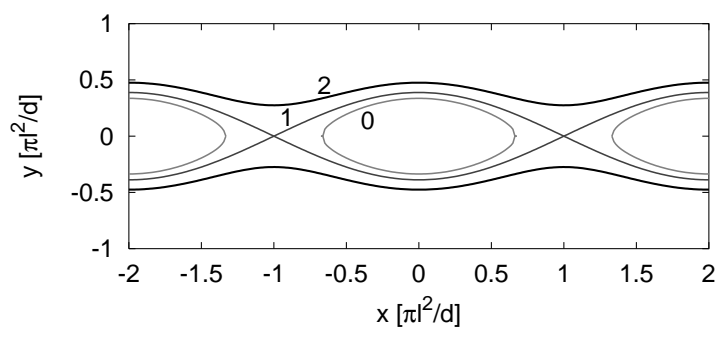

FIG. 2: Real-space semiclassical trajectories of electron in magnetic field. $0-\operatorname{closed}\left(E_{F}<2|t|\right), 1-\operatorname{critical}\left(E_{F}=2|t|\right)$, 2 - open $\left(E_{F}>2|t|\right)$. The closed trajectories are elongated in the direction parallel to the wires by a constant factor $\sqrt{m_{y} / m}$ and due to non-parabolicity of the cosine band (Eq. 1). 
So as to be able to compare the SC and QM predictions let us now examine the densities of states. Note that by comparing the zero-field DOS of our system (for $E \ll 2|t|$, i.e. in the region of quantized orbits and 2Dlike behaviour) with the zero-field DOS of a free 2DES $\left(g(E)=2 m_{\mathrm{eff}} / \pi \hbar^{2}\right.$ including spin) we may deduce the modulation-influenced effective mass $m_{\text {eff }}$. This in turn determines the quantization condition $E_{F}=\hbar \omega_{\mathrm{eff}}\left(n+\frac{1}{2}\right)$ and thus all SC predictions can be made using the zerofield DOS of the system only. Based on the spectrum (Eq. 1) we can compute the zero-field DOS analytically

$g_{0}(E)=\left\{\begin{array}{l}\frac{4}{(2 \pi)^{2}} \sqrt{\frac{2 m}{\hbar^{2}|t| d^{2}}} \cdot \frac{1}{\sqrt{\xi}} K(1 / \sqrt{\xi}), E>2|t| \\ \frac{4}{(2 \pi)^{2}} \sqrt{\frac{2 m}{\hbar^{2}|t| d^{2}}} \cdot K(\sqrt{\xi}),-2|t|<E<2|t|\end{array}\right.$

where

$$
\xi=\frac{1+E / 2|t|}{2}
$$

including the factor of two for spin (the dotted line in Fig. 3). $K$ is the full elliptic function $K(k)=F\left(\frac{\pi}{2}, k\right)=$ $\int_{0}^{\pi / 2}\left(1-k^{2} \sin ^{2} \varphi\right)^{-1 / 2} \mathrm{~d} \varphi$, we recall 17 that $K(0)=\pi / 2$, $K(1)=\infty$.

Let us focus on the weak-field case first $\left(\hbar \omega_{\text {eff }} \ll 2|t|\right)$ and discuss the influence of magnetic field $B$ on the continuous spectrum (Eq. 1), i.e. we try to estimate the DOS in magnetic fields without any calculation. On one hand, Landau levels (LLs) appear for $E_{F} \ll 2|t|$ (2Dlike paraboloid band structure, modified effective mass $m_{y}$ ). If the thermal energy is comparable to the Landau level separation $\left(k_{B} T \approx \hbar \omega\right)$ the DOS becomes oscillatory (leading to $\mathrm{SdH}$ oscillations) but approaches the zero-field DOS (see also Fig. 3). In other words, the oscillations missing in the zero-field DOS are exactly reproduced by the SC quantization condition (closed trajectories, $\left.E_{F}<2|t|\right)$ which is fulfilled just when $E_{F}$ lies in the middle between two LLs.

On the other hand, narrow gaps open in the continuous spectrum for $E_{F} \gg 2|t|$ due to the slight corrugation (by the cosine term) of the almost 1D-like parabolictrough band structure. Numerical calculations (using model from Sec. III) show that these gaps are narrow enough to disappear due to thermal broadening and the zero-field DOS matches the non-zero-field DOS perfectly. The SC approach relying on non-quantized open trajectories $\left(E_{F}>2|t|\right)$ and predicting non-oscillatory magnetoresistance will therefore be successful once again.

The picture is considerably different for strong magnetic fields $\left(\hbar \omega_{\text {eff }} \gtrsim 2|t|\right)$ at which the cyclotron radius $R_{c}$ approaches $d$. The numerically computed DOS shows no similarity to the zero-field DOS (Fig. 3 again). The non-zero-field DOS rather resembles $g(E) \propto 1 / \sqrt{E}$ of a single quantum wire (suppression of tunnelling between two neighbouring wires when classical cyclotron radii become comparable to $d$ ) with gaps both for $E<2|t|$ and

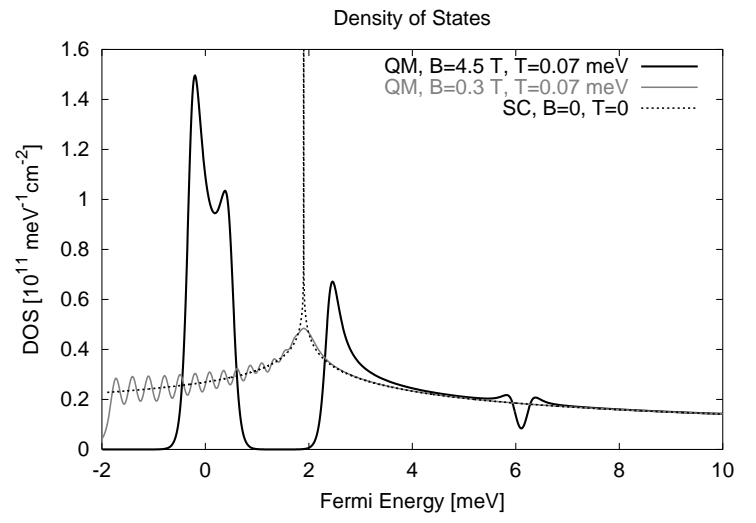

FIG. 3: The density of states for $B=0$ (dotted) and thermally broadened $(T \approx 1 \mathrm{~K})$ density of states for low field ( $\hbar \omega_{\text {eff }} \ll 2|t|$ or $\alpha \ll 1$, solid grey line) and high field (solid black line).

$E>2|t|$ owing to the tunnelling between the wires. Therefore we expect oscillatory magnetoresistance for both closed and open SC trajectories on contrary to the $\mathrm{SC}$ predictions (magnetic breakdown in the SC theory).

The gaps occur at the boundary of the first magnetic Brillouin zone (1MBZ, see the section III) and result from the periodicity of the dispersion relation in $k_{x}$ which in turn reflects the invariance of the QM Hamiltonian to magnetic translation 18 (this is what remains from the full translational symmetry in the $x$-direction after switching on the magnetic field). Magnetic breakdown can be included also in the SC picture throuph tunnelling between two open or two closed trajectoried了 (see Fig. 2). This is an ad hoc assumption though.

The failure of the SC approach for strong magnetic fields is apparent also in another context. Once the Fermi level is set and the type of trajectory is determined, the same behaviour (either 2DES-like $\mathrm{SdH}$ oscillations or 1DES-like no oscillations) is predicted for all magnetic fields. However, it is clear even on the SC level that if the cyclotron radius $R_{c}=k_{F} \ell^{2}$ ( $k_{F}$ is the Fermi wavevector, $E=\hbar^{2} k_{F} / 2 m$ ) becomes comparable to $d$, tunnelling between the wires is suppressed and the system ought to switch to a quasi-1D regime.

The QM approach is suitable for both low and strong magnetic fields. Moreover, it opens up the way to quantitative calculations of the magnetoresistance and thus to data directly comparable to the experiments.

\section{MODEL}

Having chosen the Landau gauge $\vec{A}=(B y, 0,0)$ our system is described by the separable Hamiltonian $(e=$ $|e|)$

$$
H=\frac{1}{2 m}\left(p_{x}+e B y\right)^{2}+\frac{1}{2 m} p_{y}^{2}+V(y),
$$


i.e. allowing to set $\Psi(x, y)=\exp (i k x) \psi(y)$ for the eigenfunctions. Our ansatz for the whole wavefunction is

$$
\Psi(x, y)=\frac{1}{\sqrt{2 \pi}} \exp (i k x) \sum_{j} a_{j}(k) \varphi(y-j d),
$$

i.e. we use the ansatz $|\psi(k, n)\rangle=\sum_{j} a_{j}(k)|j\rangle$ for $\psi(y)$ ( $n$ is the Landau index in the spectrum of (5) or (3) for a given $k$ ) where $|j\rangle$ is the ground state localized in the $j-$ th well of the potential (corresponds to the Wannier state of the $B=0$ case). We have thus limited our model just to the lowest band in the modulation direction by this ansatz. The Fermi level lies always deep below the top of the modulation potential in our calculations.

Next we use the tight-binding approximation (i.e. $\left.\langle i \mid j\rangle=\delta_{i, j},\langle i|H| j\rangle=t \delta_{i, j \pm 1}, t<0\right)$ and obtain the Hamiltonian matrix elements (see also Wulf et al 19 )

$$
H_{i j}=\frac{\hbar^{2}}{2 m} \cdot K^{2}((k / K)+i)^{2} \delta_{i, j}+t \delta_{i, j \pm 1}, \quad K=d \frac{e B}{\hbar} .
$$

In our model the real physical system is thus represented by the parameters $t$ (hopping) and $d$ (period) for the structure and of course $B$ for the magnetic field.

Note that (up to the scaling of $k$ and energy) the problem (5) effectively depends only on the single parameter $\alpha$ : Eq. 同 can be written in dimensionless form

$$
\begin{gathered}
H_{i j}=|t|\left[\alpha^{2}((k / K)+i)^{2} \delta_{i, j}-\delta_{i, j \pm 1}\right], \\
\alpha^{2}=\frac{e^{2} B^{2}}{m} \cdot \frac{d^{2}}{2|t|}=\left(\frac{\hbar \omega_{\mathrm{eff}}}{2|t|}\right)^{2} .
\end{gathered}
$$

If we now assume the system to be infinite in the $y$ direction the matrix problem (5) is mathematically equivalent to the Mathieu equation

$$
-\frac{\hbar^{2}}{2 m} \psi^{\prime \prime}(x)-\psi(x) 2|t| \cos K x=E \psi(x)
$$

i.e. 1D Schrödinger equation with potential $W(x)=$ $-2|t| \cos K{ }^{T}$ The detailed description of this model can be found in 20 .

This allows us to determine the spectrum of (5) qualitatively, and therefore also the DOS, even before we carry out the numerical calculations. Let the magnetic field be weak at first $(\alpha \ll 1$, see explanation below in this paragraph). Bands $E(k)$ should appear (as a consequence of the Bloch theorem) and we may limit ourselves to $1 \mathrm{MBZ}$, the first "magnetic Brillouin zone" $k \in\left(-\frac{1}{2} K, \frac{1}{2} K\right)$. For $E \ll 2|t|$ we expect those bands to be equidistantly spaced and narrow. The former follows from $W(x) \approx 2|t|\left(-1+\frac{1}{2} K^{2} x^{2}\right)$ near the potential minimum (the condition $\alpha \ll 1$ means just that the spacing of such states is $\ll 2|t|)$, the latter is due to the smallness of the overlap of low-lying states in two neighbouring wells of $W(x)$. In contrast, for $E \gg 2|t|$ we expect the spectrum to be almost like the one of $1 \mathrm{D}$ free electrons $E(k)=\sum_{i} \hbar^{2}(k+i K)^{2} / 2 m$. The underlying non-constant potential $V$ will manifest itself in the gaps which open at the Brillouin zone boundaries $\left(k= \pm \frac{1}{2} K\right)$.

Let us now translate this analysis into terms of the original problem 5 . Consider a fixed magnetic field (or constant $\alpha$ ). For low energies we obtain nearly equidistant and sharp Landau levels (free 2D electron gas in magnetic field). At high energies we get a sum of almost 1D densities of states, i.e. independent quantum wires (where $B$ plays no role). The transition occurs around $E=2|t|$. This agrees with the semiclassical theory except for the narrow gaps in the continuous quasi-free1D-electron part of the spectrum. These indicate the magnetic breakdown for quantizing magnetic fields.

Secondly, we focus on strong magnetic fields $(\alpha \gg 1)$. On one hand, the states of Eq. T below $2|t|$ will become more widely spaced and even the lowest state will no longer have $E \ll 2|t|$. We then expect even the lowest LB to be broad (solid black curve at Fig. 3) because its energy lies in an intermediate region between $E \ll 2|t|$ and $E \gg 2|t|$. On the other hand the states with $E>$ $2|t|$ which approach the free electron states (and have a nearly free-electron parabolic spectrum) will have wider gaps (the smaller the lattice constant of a crystal, the wider the gaps).

We may now compare the DOS being output from our model with the experimentally measured resistance, see Fig. 6. In the experiments, the Fermi energy was adjusted by applying a gate voltage $U_{g}$ and we assume that $E_{F}$ remains constant while magnetic field is swept.

Experiments showed that the gate voltage is proportional to the concentration of electrons ( $N$, see Sec. IV). The proportionality constant is also approximatelly equal to the capacity of a parallel-plate capacitor corresponding to the gated structure. We took the concentration to be equal to the zero-field concentration and computed the corresponding Fermi energy, neglecting the localization effects. Looking at Fig. A we see the gaps in the DOS matching very well with the straight lines of magnetoresistance extrema which justifies this $U_{g} \leftrightarrow E_{F}$ model of ours.

We can see in the Fig. 6 that varying the magnetic field and keeping the Fermi energy (or $U_{g}$ ) constant and low the system behaves like an almost free 2D electron gas (sharp LLs, equidistant in $1 / B$ ) with modified effective mass $\sqrt{m m_{y}}$ (as predicted by SC theory). At intermediate $E_{F}$ however, we observe that the narrow bands become broad at high $B$ indicating an effective 2D to $1 \mathrm{D}$ transition (when cyclotron radii become smaller than the modulation period). In contrast, the SC theory states that once the Fermi energy is set, a magnetic field cannot change the dimensionality of the system. When $E_{F}$ is high the quantum wires are decoupled, but there still open gaps in the continuous spectrum (reflecting the tunneling between the open SC orbits).

The lighter region around $B \approx 11 \mathrm{~T}$ and $U_{g} \approx 0.45 \mathrm{~V}$ in the experimental data (Fig. Abelow) suggests that our model works no longer for strong magnetic fields $(\alpha \gg$ 1 ) and low Fermi energies (even the lowest LB nearly 


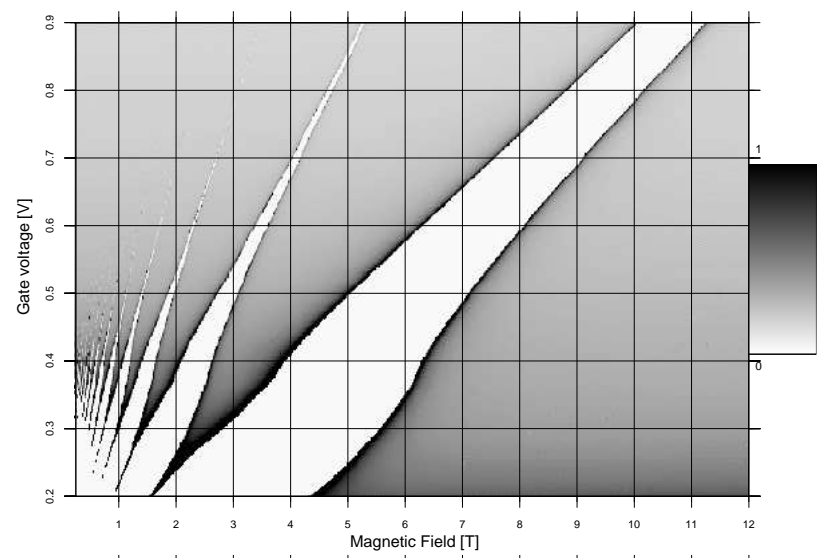

Here $\hat{v}_{x, y}$ stand for the velocity operator components, $\delta_{\Gamma}(E-\hat{H})=-(1 / 2 \pi i)\left(\hat{G}^{+}(E)-\hat{G}^{-}(E)\right)$ and $\hat{G}^{ \pm}(E)=$ $(E-\hat{H} \pm i \Gamma)$. Isotropic scattering by a random impurity potential is taken into account by means of the complexvalued self-energy $\Sigma=\Delta+i \Gamma$ and we neglect its real part. In the self-consistent Born approximation and assuming $\Gamma(E, B)$ to be small compared to the level separation (typically $\hbar \omega_{\text {eff }}$ ) we can express components of $\sigma$ by means of the DOS $g(E)$ and matrix elements of $y$ $(\omega=e B / m)$

$$
\begin{aligned}
& \sigma_{x x}(E)=\frac{2}{\pi \Gamma d} \cdot \frac{e^{2}}{h} \cdot \frac{\operatorname{sgn}(g(E))}{g(E)}+ \\
& \frac{4 \pi \Gamma}{d} \cdot(\hbar \omega)^{2} \cdot \frac{e^{2}}{h} \cdot g(E) \sum_{n^{\prime} \neq n}\left(\frac{\left\langle\psi\left(k, n^{\prime}\right)|y| \psi(k, n)\right\rangle}{E\left(k, n^{\prime}\right)-E(k, n)}\right)^{2} \\
& \sigma_{y y}(E)=\frac{4 \pi \Gamma}{d} \cdot \frac{e^{2}}{h} g(E) \sum_{n^{\prime} \neq n}\left(\left\langle\psi\left(k, n^{\prime}\right)|y| \psi(k, n)\right\rangle\right)^{2} \\
& \sigma_{x y}(E)=e \frac{\partial N(E)}{\partial B}+ \\
&+\frac{4 \pi \hbar \omega}{d} \cdot \frac{e^{2}}{h} g(E) \sum_{n^{\prime} \neq n}\left(\left\langle\psi(k, n)|y| \psi\left(k, n^{\prime}\right)\right\rangle\right)^{2}
\end{aligned}
$$

(including spin degeneracy). The symbolic expression $\operatorname{sgn}(g(E)) / g(E)$ in the first term of $\sigma_{x x}$ indicates that this term vanishes if $g(E)=0$. Just to get simpler formulae, the additional assumption has been made that there are merely two points at the Fermi level $E=E(k, n)$ within the $1 \mathrm{MBZ}$. We reproduced the older results of Wulf et al 19 where the $\Gamma$-independent $\sigma_{x y}(E)$ was calculated within the same model but in a formally different way (Eqs. 5,6 in 21).

Note the structure of the components of $\sigma$ : in genempty). This condition matches the situation when the cyclotron radius is much smaller than $d$ and it means that our tight-binding model is inappropriate for magnetic fields strong enough to create LLs within one quantum wire (so that even the width of wires will be large enough for the electrons to behave as a 2DES inside one wire). These are, however, rather extreme conditions for the experiments shown in Fig. 4 .

\section{TRANSPORT: KUBO FORMULA}

Knowing the eigenfunctions to the matrix problem 5 we pay use the linear response theory (Kubo formula, see e.g.21) to compute the conductivity tensor components (per system area $A$ )

$$
\begin{aligned}
\sigma_{i i}\left(E_{F}\right)= & \frac{\pi \hbar e^{2}}{A} \operatorname{Tr}\left[\hat{v}_{i} \delta_{\Gamma}\left(E_{F}-\hat{H}\right) \hat{v}_{i} \delta_{\Gamma}\left(E_{F}-\hat{H}\right)\right] \\
\sigma_{x y}(E)= & \frac{i \hbar e^{2}}{A} \cdot \frac{1}{2} \operatorname{Tr}\left[\hat{v}_{x} \hat{G}^{+}(E) \hat{v}_{y} \delta_{\Gamma}(E-\hat{H})-\right. \\
& \left.\hat{v}_{x} \delta_{\Gamma}(E-\hat{H}) \hat{v}_{y} \hat{G}^{-}(E)\right]+e \frac{\partial N(E)}{\partial B}
\end{aligned}
$$
eral, all the terms (except for the $\partial N / \partial B$ term in $\left.\sigma_{x y}\right)$ are products of the $\operatorname{DOS}(g)$ and some matrix elements of $\hat{y}$. There are two contributions to the conduction parallel to the wires $\left(\sigma_{x x}\right)$ : the first term (proportional to $1 / g$ ) originates from the diagonal matrix elements $\delta\left(k-k^{\prime}\right) \hbar k /(e B)+\left\langle\psi\left(k^{\prime}, n\right)|\hat{y}| \psi(k, n)\right\rangle \propto$ $\left\langle\psi\left(k^{\prime}, n\right)\left|\hat{v}_{x}\right| \psi(k, n)\right\rangle=(1 / \hbar)(\mathrm{d} E / \mathrm{d} k) \delta\left(k-k^{\prime}\right) \propto 1 / g$. It corresponds to the classical conductivity of a wire (open electron trajectories) or in other words it does not vanish owing to the unilateral modulation of the system: without modulation (free 2DES) the DOS comprises of deltapeaks (sharp LLs) and $(\operatorname{sgn} g) / g \rightarrow 0$. For $E<2|t|$, we obtain from our calculations LBs with non-zero width and this reflects tunnelling between two closed orbits in the SC picture (see Fig. 2), i.e. indicates a deviation from the quasi-2D behaviour. This contribution to conductivity is being suppressed by the impurity scattering (by $1 / \Gamma$ ) or in other words, persists even if there is no impurity scattering at all.

The conduction perpendicular to the wires $\left(\sigma_{y y}\right)$ and the second term of $\sigma_{x x}$ reflect the inter-LB transitions and appear thus due to impurity scattering (they are proportional to $\Gamma$ ). These contributions might be viewed as 
a consequence of tunnelling between the open SC orbits. There is no requirement that $\langle\psi(n, k)|y| \psi(m, k)\rangle=0$ for $|n-m|>1$ as in the limit of weak modulation, but we found these matrix elements to be decaying rapidly with growing $|n-m|$. In other words, inter-LB scattering occurs dominantly between neighbouring LBs.

The Hall conductivity does not depend on the scattering in the leading order at all.

Let us now concentrate on the issue of impurity scattering. Following 182223 we used an ansatz of self-energy depending only on energy (and $B$ ). This leads to a selfconsistent equation

$$
\Sigma(E) \propto \operatorname{Tr} \frac{1}{E-H-\Sigma(E)}
$$

where the proportionality constant describes the strength of the impurity scattering. This equation yields24 25 the well-known result

$$
\Gamma^{2}=\frac{1}{2 \pi} \hbar \omega \frac{\hbar}{\tau}
$$

for free $2 \mathrm{DES}$ (i.e. no modulation) and short-range scattering potential. $\tau$ is the relaxation-time in the zero magnetic field case (as in the Drude theory). In our calculations we used this (i.e. $\Gamma=\gamma \sqrt{B}$ ) as a phenomenological ansatz which has already proven to be useful in explaining the magnetoresistance data by long-period superlattices 2627. Surprisingly enough, even this simple ansatz provides a very good qualitative agreement with the experimental data and makes the results of the calculations depend on the fitting parameter $(\gamma$, scattering strength) in a very simple way.

In order to obtain data comparable to experiments, we need to express the resistivity tensor components

$$
\varrho_{y y}=\frac{\sigma_{x x}}{\sigma_{x x} \sigma_{y y}+\sigma_{x y}^{2}}, \quad \varrho_{x y}=\frac{\sigma_{x y}}{\sigma_{x x} \sigma_{y y}+\sigma_{x y}^{2}} .
$$

A remarkable and important point of these formulae is that if scattering is weak (i.e. the inter-LB term $\propto \Gamma$ in $\sigma_{x x}$ may be neglected), the denominators do not depend on $\Gamma$.

Due to the particular sample geometry a two-point measurement is performed and thus the experimentally accessible quantity is some mixture of $\varrho_{y y}$ and $\varrho_{x y}$. It seems plausible to assume that the voltage drop measured is a constant linear combination of the transversal and longitudinal voltage and thus the measured resistance is

$$
R=c \varrho_{y y}+\varrho_{x y}
$$

with some dimensionless constant geometrical factor $c$. Thus if scattering is weak, the magnetoresistances to be compared to experimental data contain a single fitting parameter $c / \gamma$. This comparison for different electron densities is shown at Fig. 5 .
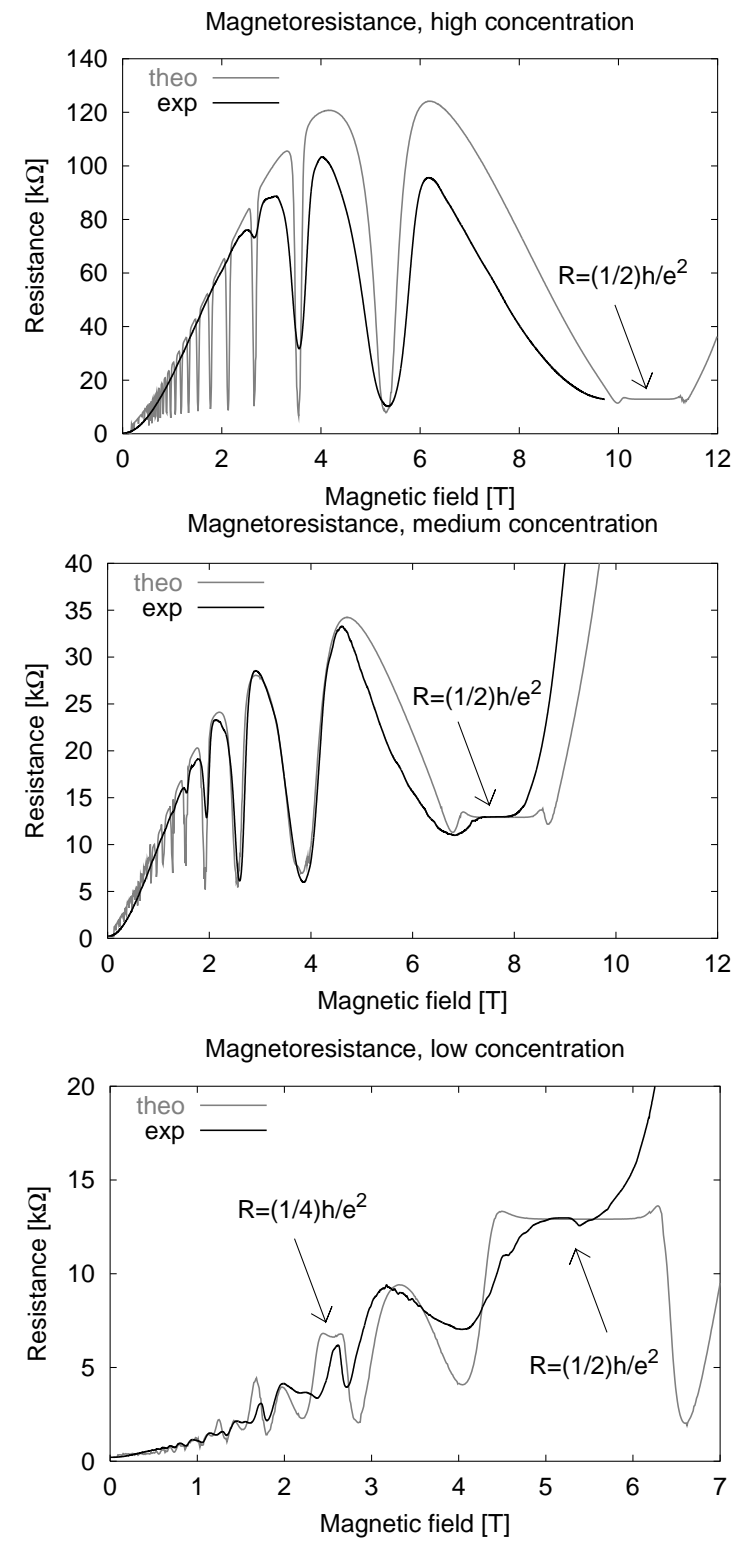

FIG. 5: Magnetoresistance. Calculations and experiment. Electron concentrations (from the top) $5.1 \times 10^{11} \mathrm{~cm}^{-2}$, $3.8 \times 10^{11} \mathrm{~cm}^{-2}, 2.65 \times 10^{11} \mathrm{~cm}^{-2}$. The lowest concentration corresponds to $E_{F} \approx 2|t|$.

\section{DISCUSSION}

Almost quantitative agreement is achieved for high concentrations (Fig. 5a,b). Exact (no fitting parameter) match between the magnetoresistance extrema in theory and experiments (which has been emphasised already on the DOS level) shows that the tight-binding model captures the essential physics in our experiments.

Note also that the magnetoresistance approaches quantized values $R=(1 / \nu) h / e^{2}$ for $E_{F}$ lying in the gap. First, this allows to determine the concentration of electrons $N$ in the system (compare filling factor of each plateau $\nu$ 
and its $B$ ). Second, it confirms our model of the relation between the gate voltage $U_{g}$ and Fermi level $E_{F}$. However, as we may see best in Fig. 5 c, there is still room for improving the model e.g. by taking the localization effects into account.

The quadratic rise of resistivity at low magnetic fields due to the diagonal component $\left(\varrho_{y y}\right)$ has been predicted within a SC mpdell and measured also at weakmodulation samples27. Our results (with single fitting parameter at Fig. 5a,b) deviate from this slightly because of the form of $\Gamma(E, B) \propto \sqrt{B}$. Assuming $\Gamma$ independent on $B$ in this region, the quadratic behaviour is reconciled. For intermediate fields the matrix elements of $\hat{y}$ start to play a role.

Another feature of the tight-binding model is the capability of explaining why the Hall plateaus show minima at high concentrations and maxima at low concentrations (Fig. 5c). Note, however, that even at this concentration $E \approx 2|t|$. Swapping the dominance of $\sigma_{x y}$ and $\sigma_{x x, y y}$ (see esp. Eq. (8) is the cause for the qualitative change of the magnetoresistance curve. Nevertheless, we are aware of the feebler match between theory and experiments at lower concentrations indicating that our ansatz for the self-energy is rather crude.

\section{CONCLUSION}

The cleaved-edge-overgrowth technology opens a new area of 2DES with short-period atomically precise unidirectional modulation for experimental studies. Such samples may be viewed as an array of coupled quantum wires which can be decoupled by applying reasonable $(\lesssim 5 \mathrm{~T})$ perpendicular magnetic field. From a theoretical point of view, these systems have a simple band structure and can be very well described by a simple tight-binding model. It was demonstrated that for weak magnetic field $\left(\hbar \omega_{\text {eff }} \ll 2|t|\right)$ the SC approach argumenting with closed and open electron trajectories is expected to be fairly good and it is good indeed. On the other hand, this approach fails for quantizing magnetic fields (magnetic breakdown in the SC terminology). The fully quantummechanical tight-binding model offers both a good way to estimate the applicability of the SC approach and a reasonable description of the system for any magnetic field unless $R_{c}=k_{F} \ell^{2} \ll d$. This model is also very intuitive on the level of DOS analysis owing to the analogy with Mathieu equation (7).

We found a good agreement between the experimental magnetoresistance and calculations based on the linear response theory even with a very simple model for impurity scattering. We expect that the match between theory and experiment may be improved by internaly-consistent treatment of scattering due to randomly distributed impurities.

\section{ACKNOWLEDGEMENTS}

One of the authors (KV) would like to acknowledge discussions with Daniela Pfannkuche and Alexander Chudnovskiy. This work has been partly supported by the Grant Agency of the Czech Republic under Grants No. 202/01/0754 and No. 202/03/0431.
* Electronic address: vybornyk@fzu.cz

$\dagger$ now with McKinsey\&Company

1 D. Weiss, K. von Klitzing, K. Ploog, and G. Weinmann, Europhys. Lett. 8, 179 (1989).

2 C. Beenakker, Phys. Rev. Lett. 62, 2020 (1989).

3 P. Středa and A. MacDonald, Phys. Rev. B. 41(17), 11892 (1990).

4 R. Gerhardts, D. Weiss, and K. v. Klitzing, Phys. Rev. Lett. 62(10), 1173 (1989).

${ }^{5}$ P. Vasilopoulos and F. Peeters, Phys. Rev. Lett. 63(19), 2120 (1989).

${ }^{6}$ P. Beton, E. Alves, P. Main, L. Eaves, M. Dellow, M. Henini, and O. Hughes, Phys. Rev. B. 42(14), 9229 (1990).

7 Q. Shi and K. Szeto, Phys. Rev. B. 53(19), 12990 (1996).

${ }^{8}$ R. Menne and R. Gerhardts, Phys. Rev. B. 57(3), 1707 (1998).

9 S. Zwerschke and R. Gerhardts, Physica E (256-258), 28 (1998).

10 S. Zwerschke, A. Manolescu, and R. Gerhardts, Phys. Rev. B. 60(8), 5536 (1998).

11 J. Müller, Phys. Rev. Lett. 68(3), 386 (1992).

12 F. Peeters and P. Vasilopoulos, Phys. Rev. B. 47(3), 1466 (1993).

13 R. A. Deutschmann, A. Lorke, W. Wegscheider, M. Bich- ler, and G. Abstreiter, Physica E 6(1-4), 561 (2000).

14 R. A. Deutschmann, W. Wegscheider, M. Rother, M. Bichler, G. Abstreiter, C. Albrecht, and J. H. Smet, Phys. Rev. Lett. 86(9), 1857 (2001).

15 R. A. Deutschmann, Two dimensional electron systems in atomically precise periodic potentials, Selected topics of Semiconductor Physics and Technology, Vol. 42 (University of Technology Munich, 2001), PhD. thesis, ISBN 3932749-42-1.

16 N. Ashcroft and N. Mermin, Solid State Physics (Saunders College Publishing, Orlando, 1976), p. 232.

17 I. Gradshteyn and I. Ryzhik, Table of Integrals, Series and Products (Academic Press, San Diego, 1980), p. 904.

18 D. Pfannkuche and R. Gerhardts, Phys. Rev. B. 46(19), 12606 (1992).

19 U. Wulf, J. Kučera, and A. MacDonald, Phys. Rev. B. 47(3), 1675 (1992).

20 S. Davidson and M. Stéşlicka, Basic Theory of Surface States (Claredon Press, Oxford, 1992), p. 41.

21 P. Středa, J. Phys. C: Solid State Phys. 15, L717 (1982).

22 C. Zhang and R. Gerhardts, Phys. Rev. B. 41(18), 12850 (1990).

23 T. Ando, A. Fowler, and F. Stern, Rev. Mod. Phys. 54, 536 (1982).

24 R. Gerhardts and J. Hajdu, Zeitschrift für Physik 245, 126 
(1971).

25 R. Gerhardts, Zeitschrift für Physik B 22, 327 (1975).

26 A. Manolescu, R. Gerhardts, M. Tornow, D. Weiss, K. von Klitzing, and G. Weinmann, Surf. Sci. 361/362, 513
(1996).

27 M. Tornow, D. Weiss, A. Manolescu, R. Menne, and K. von Klitzing, Phys. Rev. B. 54(23), 16397 (1996). 